\title{
Exploring the Possibilities: Earth and Space Science Missions in the Context of Exploration
}

\author{
Barbara Pfarr \\ NASA/Goddard Space \\ Flight Center (GSFC) \\ Greenbelt, MD 20771 \\ 301-286-6663 \\ Barbara.pfarr@nasa.gov
}

\author{
Michael Calabrese \\ SGT Inc. \\ NASA/GSFC \\ Greenbelt, MD 20771 \\ 301-286-7287 \\ michael.calabrese@gsfc.nasa.gov
}

\author{
James Kirkpatrick \\ American Astronautical Society \\ 6352 Rolling Mill Place, \\ Ste 102 \\ Springfield, VA 22152 \\ 703-866-0020 \\ jkirkpatrick@astronautical.org
}

\author{
Jonathan T. Malay \\ Lockheed Martin Corp. \\ 1550 Crystal Dr, Ste 300 \\ Arlington, VA 22202 \\ 703-413-5816 \\ jon.malay@lmco.com
}

Abstract - According to Dr. Edward J. Weiler, Director of the Goddard Space Flight Center, "Exploration without science is tourism". At the American Astronautical Society's $43^{\text {rd }}$ Annual Robert H. Goddard Memorial Symposium it was quite apparent to all that NASA's current Exploration Initiative is tightly coupled to multiple scientific initiatives: exploration will enable new science and science will enable exploration. NASA's Science Mission Directorate plans to develop priority science missions that deliver science that is vital, compelling and urgent. This paper ${ }^{1,2}$ will discuss the theme of the Goddard Memorial Symposium that science plays a key role in exploration. It will summarize the key scientific questions and some of the space and Earth science missions proposed to answer them, including the Mars and Lunar Exploration Programs, the Beyond Einstein and Navigator Programs, and the Earth-Sun System missions. It will also discuss some of the key technologies that will enable these missions, including the latest in instruments and sensors, large space optical system technologies and optical communications, and briefly discuss developments and achievements since the Symposium. Throughout history, humans have made the biggest scientific discoveries by visiting unknown territories; by going to the Moon and other planets and by seeking out habitable words, NASA is continuing humanity's quest for scientific knowledge.

\section{TABLE OF CONTENTS}

1. INTRODUCTION.....................................................1

2. KEYNOTE ADDRESS.................................................1

3. SCIENCE GOALS AND OBJECTIVES .....................2

4. SCIENCE QUESTIONS..............................................3

5. ROBOTIC LUNAR EXPLORATION .........................4

6. ROBOTIC MARS EXPLORATION............................4

7. EXPLORING THE UNIVERSE ................................5

8. KEY TECHNOLOGIES .............................................5

9. SOLAR AND EARTH SCIENCE ...............................6

10. CONCLUSION ......................................................6

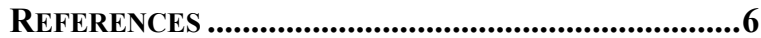

BIOGRAPHIES …........................................................

1

${ }^{1}$ U.S. Government work not protected by U.S. copyright.

2 IEEEAC paper \#1190, Version 2, dated Dec 21,, 2005

\section{INTRODUCTION}

The American Astronautical Society hosted the $43^{\text {rd }}$ annual Robert H. Goddard Memorial Symposium March 29 and 30, 2005 at the Greenbelt Marriott Hotel in Greenbelt, Maryland. Each year's conference has a different theme reflecting the interests and concerns of the astronautical community at the time. The 2004 Symposium occurred only months after the President announced his vision for space exploration and so the theme of the symposium was, fittingly "Exploration: to the Moon and Beyond". This year, in recognition of the organizations in the Washington DC area that engage in and support scientific research, including the Goddard Space Flight Center and the National Oceanographic and Atmospheric Administration (NOAA), the theme chosen was "Earth and Space Science: Exploring the Possibilities". This paper will discuss the highlights of this conference and some of the activities that have occurred since March. Readers interested in viewing the PowerPoint presentations of most of the speakers will find then located on the American Astronautical Society website. [1]

\section{KEYNOTE ADDRESS}

Dr. Jim Garvin, NASA Chief Scientist, opened the Symposium with a keynote address enthusiastically supporting the idea that "Exploration is Science" with several important themes.[2]

The first theme is that exploration throughout history has been a catalyst for scientific discovery - from the time of Columbus, through Lewis and Clark, the Wright Brothers and up to Apollo. Garvin argues, as do many others, that humans "onsite" enable discovery in a way and at a level that is far beyond even the most sophisticated robots. Ed Weiler, in his Maryland Roundtable talk, used a very colorful example - we are currently searching extra solar planets for chemical signatures of life, but the real proof will come when we travel to a planet, to its nighttime side, and see the lights of the cities come on! Garvin further 


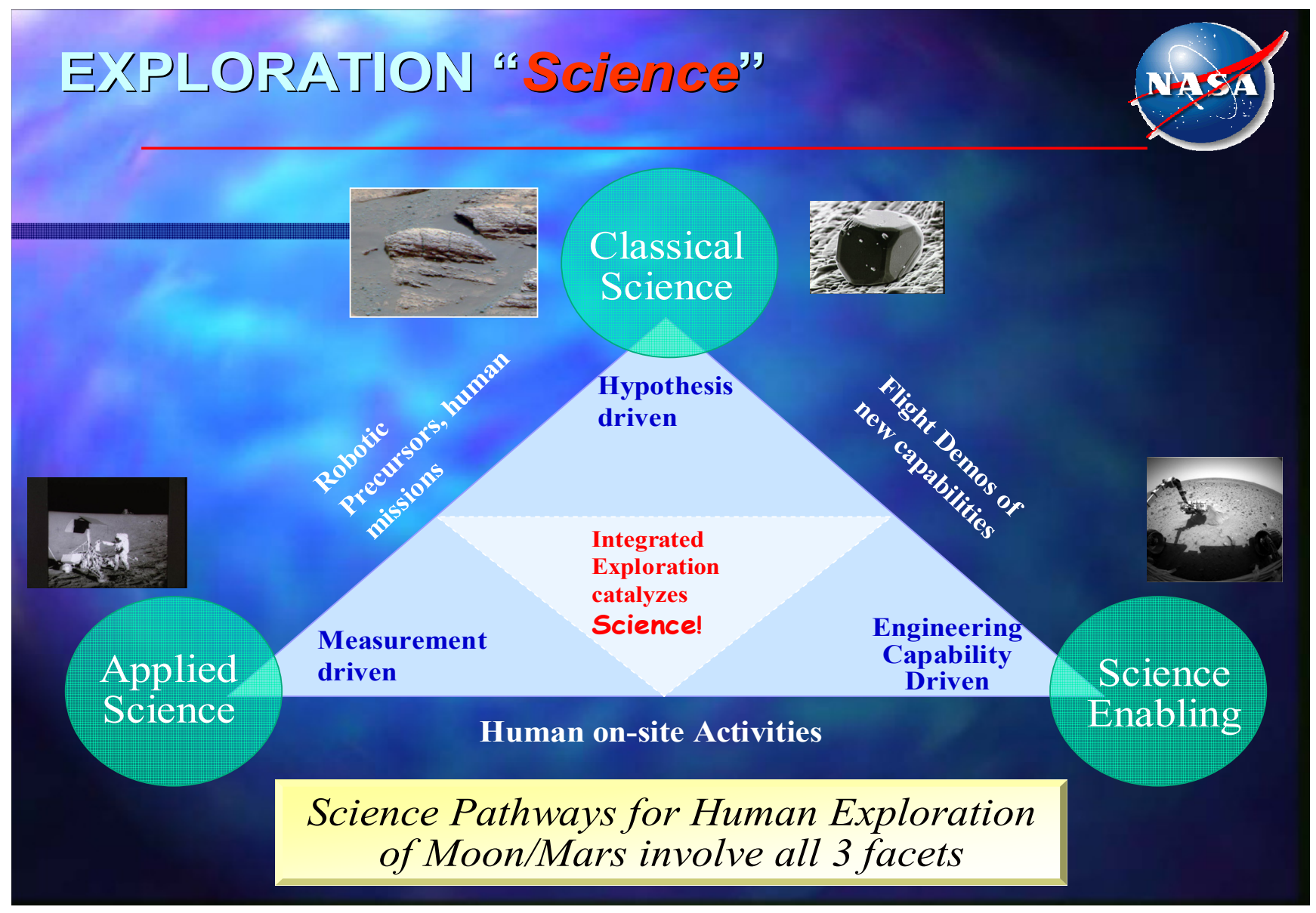

argued that just getting there isn't enough - that immense science payoff occurs when exploration leads to actual landings, as in the Apollo lunar landings and the Martian probe landings. Even further payoff is tied to mobility technology such as the Martian rovers. According to Garvin, "no mobility, no break-through science!" $\mathrm{He}$ proved this by discussing at length and with breathtaking images the geological breakthroughs that have been made possible by the Spirit and Opportunity rovers.

The second theme is that science should adapt to the Exploration Initiative as a new context. This new context has a unique set of problems and opportunities, and science can play a role in addressing these. This includes problems such as detecting and protecting from solar radiation, and choosing optimal landing sites.

A third theme is that exploration will actually enable science that wouldn't be done otherwise, or at least not to the same extent - science such as detection of space weather from the moon, and the search for life's origins on the Martian surface. Garvin's definition of "Exploration Science" is therefore a threefold blend of hypothesis driven classical science, measurement driven applied science, and engineering capability driven science enabling.

\section{SCIENCE GOALS AND OBJECTIVES}

Mary Kicza, then NASA's Associate Deputy Administrator for systems integration, stated, "The fundamental goal of the Vision for U.S. Space Exploration is to advance U.S. scientific, security, and economic interests through a robust space exploration program."[3] Our national objectives include "Implement a sustained and affordable human and robotic program to explore the solar system and beyond" and "Study the Earth system from space and develop new space-based and related capabilities for this purpose". Our NASA objectives under the first national objective include:

1) Undertake robotic and human lunar exploration to further science, and to develop and test new approaches, technologies, and systems to enable and support sustained human and robotic exploration of Mars and more distant destinations.

2) Conduct robotic exploration of Mars to search for evidence of life, to understand the history of the solar system, and to prepare for future human exploration.

3) Conduct robotic exploration across the solar system for scientific purposes and to support human exploration. In particular, explore Jupiter's moons, 
asteroids and other bodies to search for evidence of life, to understand the history of the solar system, and to search for resources.

4) Conduct advanced telescope searches for Earth-like planets and habitable environments around other stars, and

5) Explore the universe to understand its origin, structure, evolution, and destiny.

The NASA objectives that follow from the second National objective include:

1) Conduct a program of research and technology development to advance Earth observation from space, improve scientific understanding, and demonstrate new technologies with the potential to improve future operational systems.

2) Explore the Sun-Earth system to understand the Sun and its effects on Earth, the solar system, and the space environmental conditions that will be experienced by human explorers, and demonstrate technologies that can improve future operational Earth observation systems.

Ms. Kicza went on to describe two large distributed efforts that NASA had undertaken to create a series of thirteen strategic and fifteen capabilities roadmaps which would then be integrated into strategic architectures. The strategic roadmaps were summarized for NASA management on May 22, 2005, presented to the National Research Council for review in June, 2005 and then finalized for publication. The capability roadmaps were also summarized on May 22, 2005 and released as "NASA Capability Roadmaps Executive Summary." Since then, NASA released the Exploration Systems Architecture Study (ESAS) report on September 19, 2005, and is pressing forward to implementation of that architecture.

\section{SCIENCE QUESTIONS}

Marc Allen, then NASA's Assistant, Associate Administrator for Strategy, Policy and International, stated that NASA's Science Mission Directorate has as its overarching purpose "Comprehensive scientific investigations into the origin, evolution and destiny of the Earth, the Solar System and the Universe"'[4]. He presented the key scientific questions to be addressed by each of the Divisions within the Directorate. For Earth Sun systems, the questions are

1) What is causing the Earth to change and how will the Earth System change in the future?

2) How does the variability of the Sun and the space environment affect life on Earth?

3) What hazards do the variable Sun and space environment present to space travelers?

For Solar System Exploration, the questions are quite simply:
1) Where do we come from?

2) Where are we going?

3) Are we alone?

In a recent talk at the Maryland Space Business Roundtable, Goddard Center Director Ed Weiler suggested that discovering the presence of other life in the Universe could be the greatest historical achievement of the coming century, if we choose to pursue it, far greater that returning to the moon or even landing humans on Mars. The questions asked by the Universe Division include:

1) What Powered the Big Bang?

2) What is the Dark Energy Pulling the Universe Apart?

3) Where do Planets Come From?

4) What Happens at the Edge of a Black Hole?

5) Where Do the Elements of Life Come From?

6) Are There Other Habitable Worlds?

Dr. Allen emphasized the success and value of NASA science by listing the science highlights for the year 2004: The Aura, Mercury Surface, Space Environment, Geochemistry and Ranging (MESSENGER) and Deep Impact satellites were launched, Stardust returned data from its encounter with Comet Wild 2, the Spitzer Space Telescope took fabulous infrared images and spectra of distant galaxies, Cassini-Huygens gave us new images and data of Saturn and its moons, the Gravity Recovery and Climate Experiment (GRACE) provided new data on ocean currents, and Genesis returned its samples of solar ions from the solar wind, albeit with a difficult landing. And of course, the Martian rovers Spirit and Opportunity have kept us all spellbound with their images and longevity. Since the Symposium in March, NASA has had the successful launch of the Mars Reconnaissance Orbiter, a mission predicted to "return more data about the red planet than all other Mars missions combined"[5] and the total success of the Deep Impact mission, which precisely impacted Comet Tempel 1 on July 4. Dr. Allen presented a chart showing the projected NASA science launches from Calendar Year (CY) 04 through CY10, showing a very healthy schedule of 8-10 launches per year through CY08, but only 3 per year in CY09 and CY10. 

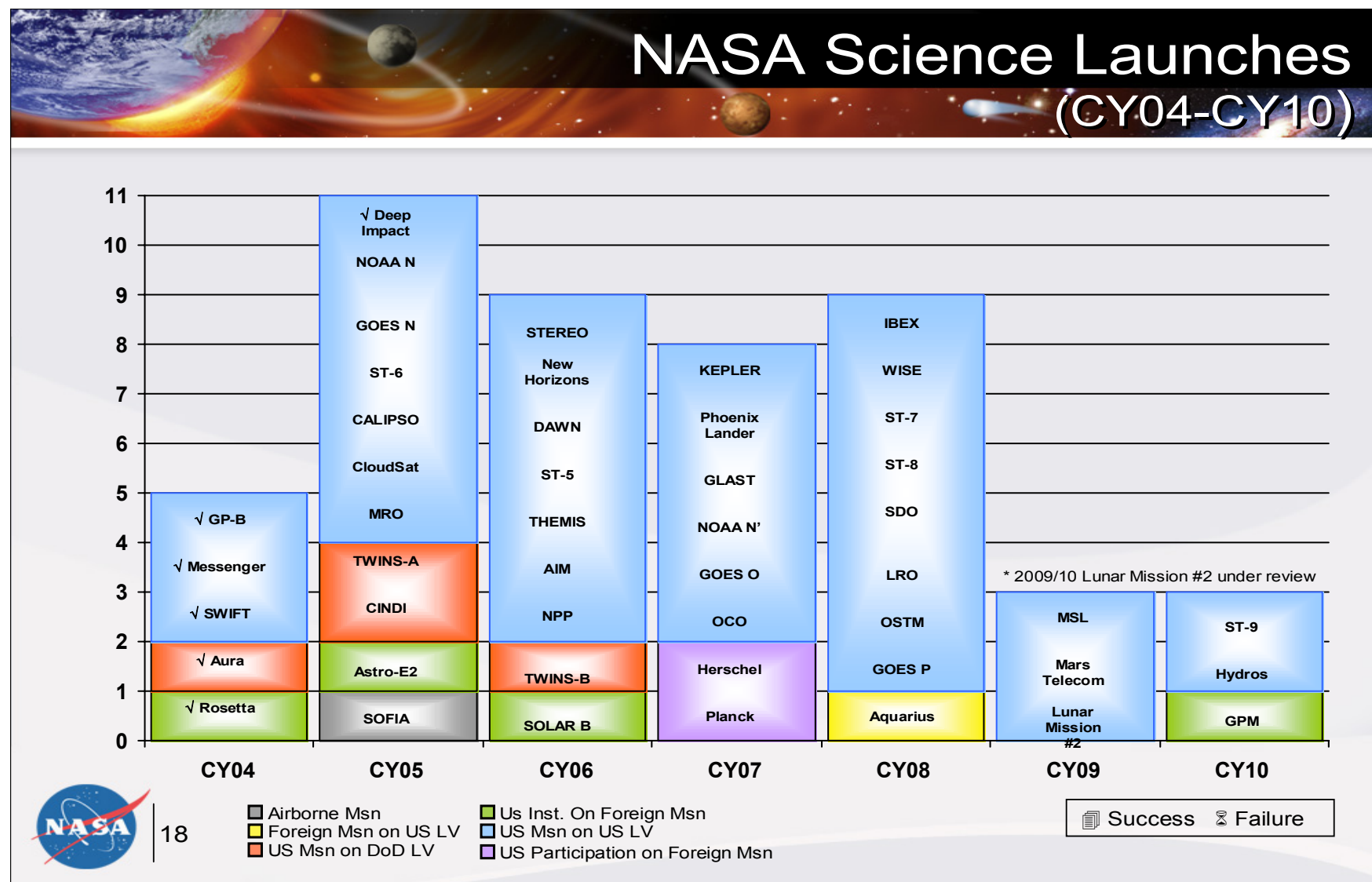

\section{ROBOTIC LUNAR EXPLORATION}

At this point, the skeptical listener at the Symposium may like everything he/she has heard, yet wonder if these bold goals of integrating science in the Exploration Initiative will really happen. So speaking next was Jim Watzin, then Program Manager of the Robotic Lunar Exploration Program (RLEP), discussing how he and his team are integrating science into the Exploration program at the current time [6]. Mr. Watzin sees robotics missions as an integral part of Exploration, to "lead the way" for the future manned missions. RLEP will support all three of the previously defined aspects of Exploration Science. The first mission, the Lunar Reconnaissance Orbiter, has six science instruments: The Lunar Orbiter Altimeter Measurement Investigation, the Lunar Reconnaissance Orbiter Camera, the Lunar Exploration Neutron Detector, the Diviner Lunar Radiometer Experiment, The Lyman-Alpha Mapping Project, and The Cosmic Ray Telescope for the effects of Radiation. These instruments do a wide range of science including imaging, altimetry, topography, spectroscopy, hyperspectral mapping, and in-situ geochronology. Potential science objectives for the following missions include:

2) Validation of water ice

3) In-situ biological sentinel experiments

4) Regolith characterization in $3 \mathrm{D}$

5) Far-side gravity

6) Landing site hazards
7) In-situ resource utilization (ISRU) experiments, and

8) Advanced bio-adaptation experiments.

Since the conference in March, the LRO mission had a successful Systems Requirements Review in August and is moving towards a Preliminary Design Review in November, and the team to manage the second mission, the Lunar Lander, was announced in October, with the goal still to launch LRO in 2008, and the Lunar Lander not long afterwards.

\section{ROBOTIC MARS EXPLORATION}

Robotic exploration of Mars was well underway long before the President articulated the new Vision for Space Exploration. Dr. Curt Niebur began his talk about the Robotic Mars Exploration Program [7] with some of the scientific successes so far: first, Mars Global Surveyor, which is on its 3rd mission extension (Oct. 2004 - Sept. 2006), proceeding well, and has been productive longer than any other spacecraft sent to Mars and has returned more images than all past Mars missions combined. The next success is the Mars Odyssey, also in an extended mission phase, which has revealed abundant water ice in the upper few feet of the surface of Mars, at high latitudes, and has also proven to be successful as a UHF relay for the Mars Exploration Rover (MER) project for data return. The 
third success so far is of course, the rovers, Spirit and Opportunity.

The Mars Exploration Program endeavors to take advantage of each and every launch window for Mars. This summer the Mars Reconnaissance Orbiter was successfully launched on its mission, with seven instruments to do climate and high resolution mineralogy and morphology at key sites. Phoenix, a scout mission planned to perform In situ investigation of volatiles, organic molecules, \& modern Martian climate, is on track to launch in 2007. Then the Mars Science Laboratory, whose mission is to explore and quantitatively assess a local region on the Mars surface as a potential habitat for life, past or present, is planned for a 2009 launch. Dr. Niebur reinforced Dr. Garvin's message that mobility on Mars has and will yield a wide range of scientific discoveries.

\section{EXPLORING THE UNIVERSE}

It is impossible to talk about exploring the Universe without talking about the Hubble Space Telescope. At the Symposium, Dr. Mike Hauser discussed the remarkable science and scientific discoveries that the Hubble has made since its launch in 1990 [8], including determination of the age of the universe, imaging proof of gravitational lensing, the evolution of galaxies, and successful searches for evidence of extra solar planets. Recently, images of the moon have been taken, to aid in landing site selection. Then Dr. Jonathan Gardner discussed the Hubble successor mission, the James Webb Space Telescope[9] and the groundbreaking science expected from it. From here we went on to look at the two other programs that will explore the Universe, Beyond Einstein and Navigator.

\section{Beyond Einstein}

The Beyond Einstein Program takes the NASA objective to "Explore the universe to understand its origin, structure, evolution, and destiny," listed above, and breaks it into 3 fundamental questions:

1) What powered the Big Bang?

2) What is Dark Energy?

3) What happens at the edge of a black hole?

As described by Dr. Nicholas White [9], The Program includes two observatories to provide breakthrough capabilities to address Beyond Einstein science: the Laser Interferometer Space Antenna (LISA) to measure gravitational waves from merging black holes and Constellation- $X$ to observe matter falling into black holes and to \& address the mysteries of dark matter and dark energy. A series of Einstein probes will focus on answering the questions:

1) How does dark energy evolve?

2) Can we detect the Big Bang? and

3) How many black holes are there?
In particular, the probe known as the Joint Dark Energy Mission (JDEM) will make measurements to determine how dark energy evolves, which is thought to comprise over $70 \%$ of the universe and appears to be causing the expansion of the universe to accelerate. The Inflation Einstein Probe will attempt to measure the polarization of the cosmic microwave background with the goal of detecting the signature of the universe's inflation.

\section{Gamma Ray Astronomy}

In the nearer term, the Gamma Ray Large Area Space Telescope (GLAST) will search for the gamma-ray signature from the decay of dark matter particles and is a precursor of these other missions, with a launch date of 2007. Since the Symposium, using data from the Swift satellite and the High-Energy Transient Explorer (HETE), scientists have solved a 35-year-old mystery of the origin of powerful, split-second flashes of light called short gammaray bursts. By analyzing the afterglow of 2 of these bursts, scientists have discovered that they arise from collisions between a black hole and a neutron star or between two neutron stars.

\section{Navigator Program}

Searching for and characterizing extra solar planetary systems is a key component of the President's 2004 Vision for Space Exploration, and the Navigator Program is attempting to do that with two planned space missions, the Space Interferometry Mission (SIM) PlanetQuest and the Terrestrial Planet Finder (TPF). As described at the Symposium [10] SIM will detect first Earth-mass objects around nearby stars, determine planetary orbits and masses, critical parameters for determining habitability, and optimize target selection for TPF. TPF-Coronagraph will make first direct detections of light from terrestrial planets and begin remote sensing characterization and search for signposts of life, while the TPF-Interferometer will extend survey to more distant stars, complete physical characterization of planets, including habitability, and ensure robust detection

capability for life.

\section{Key TECHNOLOGIES}

Time limitations at the Symposium meant we were able to address only a very small sample of the technologies that are necessary to enable the science defined above. In the area of instruments and sensors, NASA technology investment in microwave instruments and sensors, multispectral imaging, lasers and LIDAR, direct sensing of fields, particles and waves, and in-situ instrumentation will enable several exploration missions.[12] Optical communications will enable dramatic improvements in science data rates and will lower the cost per byte of data returned.[13] The technologies needed to image extra solar 
planets include Fizeau interferometry, fast, active control of mirror segments, lightweight replicable optics, instantaneous acquisition phase shifting interferometry, and advanced optical modeling.[14] With Project Columbia, NASA has one of the most powerful supercomputing facilities in the world, which has many uses for exploration including engineering and scientific data modeling.[15] NASA is also exploring sensor web technologies to allow for improved collaboration between scientific satellites and ground systems.[16]

\section{SOLAR AND EARTH SCIENCE}

Dr. Tom Moore addressed the oft-asked question "What role can Solar and Earth Science play in exploration?" One answer is "to understand the Sun and its effects on Earth, the solar system, and the space environmental conditions that will be experienced by human explorers, and demonstrate technologies that can improve future operational systems"[17]. Moore calls his science astroplanetology, a combination of the disciplines of planetology, including nonlinear dynamics, climatology, atmospheric electricity, atmospheric physics, and gravitation, with the disciplines of astrobiology - molecular biophysics, health physics, plasma astrophysics, and solar and helio-physics, and the classical disciplines of Geology, oceanography, meteorology and zoology. All of this must be synthesized in order to fully understand and predict the hazards of the environments of the future human explorers. We must protect our astronauts on their journeys. Systemwide operational space weather forecasting will require a better understanding of Solar CMEs, shocks, solar energetic particle events, atmospheric drag structure, radiation belts variability, galactic cosmic ray flux, lunar dust levitation and transport, and Martian atmosphere dynamics. One direct application of understanding atmospheres and winds for robotic missions as well is aerocapture/aerobreaking, useful for landing on Mars and other planets and moons, despite currently large variations from the predicted models. There are several future NASA Earth Science missions in formulation or development to study the Sun-Earth system[18], with benefits for both life on Earth as well as exploration. These include several to study clouds in various ways: The Aeronomy of Ice in the Mesosphere (AIM), Cloud Aerosol Lidar and Infrared Pathfinder Satellite Observations (Calipso) and Cloudsat. Aquarius will study global sea surface salinity, while the Ocean Surface Topography Mission (OSTM) will measure sea surface height. The Time History of Events and Macroscape Interactions during substorms (THEMIS) will study the onset of magnetic storms within the tail of the Earth's magnetosphere, and the Interstellar Boundary Explorer (IBEX) will detect the shock front at the edge of the Solar System.

\section{CONCLUSION}

This paper has only covered a fraction of the information presented during the two day Goddard Memorial Symposium, "Earth and Space Science: Exploring the Possibilities". This paper has described how science is intrinsically part of the goals and objectives for the Space Exploration Initiative, how Space Exploration not only allows for science, but actually requires it, and how the scientific programs and enabling that support Exploration are moving forward in this Era of Exploration.

\section{REFERENCES}

[1] American Astronautical Society Web site http://www.astronautical.org

[2] Jim Garvin, "A Vision of Exploration: Science Possibilities", 43rd Robert H. Goddard Memorial Symposium, March 2005.

[3] Mary Kicza, "Strategic Management in the Transformed NASA”, 43rd Robert H. Goddard Memorial Symposium, March 2005.

[4] Marc Allen, "The Science Mission Directorate", 43rd Robert H. Goddard Memorial Symposium, March 2005.

[5] Mars Reconnaissance Orbiter Web site http://www.nasa.gov/mission_pages/MRO/main/index.html

[6] Jim Watzin, "An Introduction to the Robotic Lunar Exploration Program (RLEP)" 43rd Robert H. Goddard Memorial Symposium, March 2005.

[7] Curt Niebur, "The Mars Exploration Program: Still Following the Water", 43rd Robert H. Goddard Memorial Symposium, March 2005.

[8] Mike Hauser, "The Hubble Space Telescope", 43rd Robert H. Goddard Memorial Symposium, March 2005.

[9] Jonathan Gardner, "James Webb Space Telescope", 43rd Robert H. Goddard Memorial Symposium, March 2005 .

[10] Nicholas White, "Beyond Einstein: From the Big Bang to Black Holes", 43rd Robert H. Goddard Memorial Symposium, March 2005.

[11] Stephen Unwin and William Danchi, "Space Interferometers", 43rd Robert H. Goddard Memorial Symposium, March 2005.

[12] Richard Barney, "Science Instruments and Sensors", 43rd Robert H. Goddard Memorial Symposium, March 2005 . 
[13] Bernard Edwards, "Optical Communications in Support of Science from the Moon, Mars, and Beyond", 43rd Robert H. Goddard Memorial Symposium, March 2005.

[14] Lee Feinberg, "Large Space Optical System Technologies at GSFC", 43rd Robert H. Goddard Memorial Symposium, March 2005.

[15] Walter Brooks, "Project Columbia: Supercomputing Resources for NASA Scientists", 43rd Robert H. Goddard Memorial Symposium, March 2005.

[16] Stephen Talabac, "Sensor Webs - Enabling Exploration and Opportunistic Science Measurements", 43rd Robert H. Goddard Memorial Symposium, March 2005.

[17] Tom Moore "Astroplanetology: The New Science of Exploration", 43rd Robert H. Goddard Memorial Symposium, March 2005.

[18] NASA Earth Sun System Missions Website http://science.hq.nasa.gov/missions/earth-sun.html

\section{BIOGRAPHIES}

Barbara Pfarr is currently an Associate Chief of the

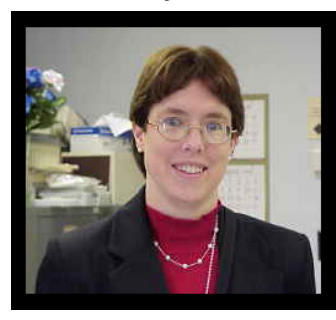
Information Systems Division of the Goddard Space Flight Center. Previously, she was head the Earth Sciences Missions Branch, which provides end-toend systems engineering for NASA programs, missions and projects and head of the RealTime Software Engineering Branch, which develops Command and Control systems and simulators. She served as the Program Chair for the AAS Goddard Memorial Symposium in March 2005. She chaired the INCOSE Systems Engineering Management Working Group during 2003. She received a B.A. in mathematics and astronomy from Smith College in 1981, a M.S in Computer Science (concentration: Artificial Intelligence) from Johns Hopkins in 1991, and a M.S. in Computer Science (concentration: Graphics) from George Washington University in 1998.

Michael Calabrese is currently a SGT, Inc. Senior Program

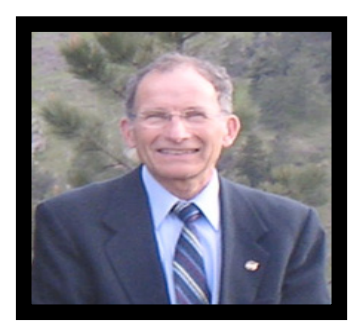
Analyst supporting the Sun-Earth Connection Program at the Goddard Space Flight Center (GSFC). His current assignments have included support of the SunSolar System Connection Strategic Roadmap Team, support of the Core Definition Phase Education and Public Outreach Plan for the New Millennium Program Space Technology-9 Solar Sail Mission, and member of the Robert H. Goddard Symposium Planning Team. Mr. Calabrese's 40 year NASA career included space science program management responsibilities at NASA GSFC and NASA Headquarters. He received a B.S. in Mechanical Engineering from Case Western Reserve University in 1960 and a Master of Public Administration from Syracuse University in 1979.

Jonathan T. Malay is the Director of Civil Space Programs

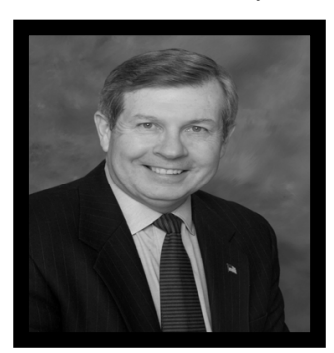
in the Washington Operations of Lockheed Martin Corp., having joined Lockheed in the spring of 2003. Prior to this, he was the Washington-based business development director for Civil Space Systems and acting head of Washington Operations for Ball Aerospace \& Technologies Corp. He is a retired Navy Surface Warfare Officer and specialist in meteorology and oceanography with twenty years of active service. In the early 1980's, he served as a visiting advisor for oceanography to the astronaut corps at NASA's Johnson Space Center. Although he was a finalist for NASA astronaut selection in 1984, he went on to serve on USS NIMITZ, at the Naval Space Command, the Office of the Oceanographer of the Navy (CNO N096), and the National Reconnaissance Office. Mr. Malay is currently Chair of the National Capital Section of the AIAA, President of the American Astronautical Society, and is a 35 year professional member of the American Meteorological Society. He is the author of a novel, Seraphim Sky, and coauthor of the National Geographic Encyclopedia of Space. He is a 1973 graduate of the U.S. Naval Academy and earned his M.S. in Meteorology from the Naval Postgraduate School in 1974.

Jim Kirkpatrick was born in Champaign, Illinois. He holds a BA in Political Science from Augustana College in Rock Island, Illinois and an MA in Human Resources Management from Pepperdine University. A retired Naval Intelligence Officer, Kirkpatrick made deployments to both

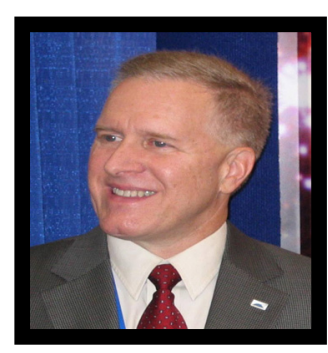

the Western Pacific and Mediterranean, and his last active duty assignment was as the Assistant to the Director of Naval Intelligence for Foreign Liaison. Jim has been the Executive Director of the American Astronautical Society since 2000. He first joined the Society in 1993 , and has served as member of the Executive Committee and Board of Directors, and held the office of Executive Vice President. Jim is an active member of St. Andrew's Episcopal Church, the Greater Springfield Chamber of Commerce, the Fairfax County Republican Committee, and the SETI Institute. 